\title{
La formación del maestro primario rural
}

Maria Luisa Ramos Grandal ${ }^{1}$

${ }^{1}$ Universidad de Artemisa. Departamento Superación de Cuadros. Calle 8C, No 713 entre 7 y campo. Consejo Popular Linconl. Municipio Artemisa. Provincia Artemisa, Cuba. mluisa@uart.edu.cu

RESUMEN. La formación del maestro primario de nivel medio que se desempeñará en escuelas rurales demanda un estrecho vínculo entre la teoría y su práctica campestre, por lo que necesita de una formación inicial investigativa que le permita apropiarse de los contenidos mínimos indispensables y flexibles que sustente el ejercicio de la función investigativa en su desempeño profesional. El objetivo de este artículo es reflexionar acerca de las posibilidades que ofrece el enfoque investigativo para el abordaje de los contenidos de la investigación educativa en el proceso de formación inicial de los maestro primario de nivel medio, con énfasis para el que se desempeñará en escuelas rurales. De la bibliografía revisada se define la formación inicial investigativa; el enfoque investigativo desde la dialéctica materialista y se explicita cómo implementarlo en el proceso formativo. La propuesta ha sido evaluada y aprobada por las autoridades del Ministerio de Educación de Cuba, se encuentra en fase de organización y diseño para su implementación en las escuelas pedagógicas del país.

Palabras-clave: Formación Inicial Investigativa, Enfoque Investigativo, Maestro Primario de Nivel Medio, Entorno Rural. 


\title{
The rural primary teacher's formation
}

\begin{abstract}
The formation of the primary school teachers in the mid-level who will work in rural schools demand a close link between theory and his/her country practice, so it is necessary an investigative initial training that allow them to acquire the essential and flexible minimum content that supports the exercise of the investigative function in their professional performance. The aim of this article is to reflect on the possibilities offered by the investigative approach to addressing the content of educational research in the process of initial training of primary teacher mid-level, with emphasis on those who will serve in rural schools. From the research literature reviewed some terms as initial training and the research approach, from dialectical materialism, are defined and it is also explained how to implement that approach in the training process. The proposal has been evaluated and approved by the authorities of the Ministry of Education of Cuba, it is in the process of organization and design for implementation in pedagogical schools.
\end{abstract}

Keywords: Initial Investigative Training, Investigative Approach, Primary School Teachers in the Mid-Level, Rural Environment. 


\section{A formação do professor do primário rural}

RESUMO. A formação do professor em nível médio que irá atuar em escolas primárias rurais de Cuba exige uma estreita ligação entre a teoria e a prática, pois é necessária uma formação inicial de pesquisa que lhe permite apropriar-se do conteúdo mínimo essencial e flexível que fundamenta o exercício da pesquisa no seu desempenho profissional. A partir dessa perspectiva, o objetivo deste artigo é refletir sobre as possibilidades oferecidas pela abordagem de investigação para abordar o conteúdo da pesquisa educacional no processo de formação inicial de professores primários de nível médio em escolas rurais cubanas. A partir da literatura científica da área, principalmente no que se refere à formação inicial e a avaliação, a abordagem desta pesquisa se fundamenta na perspectiva do materialismo histórico e dialético, no intuito de auxiliar a entender a possibilidade de implementação da abordagem de investigação no processo de formação docente. Diante disso, foi constatada que essa proposta, que está em processo de organização, foi avaliada e aprovada pelas autoridades do Ministério da Educação de Cuba, e que há um projeto para implementação dessa proposta nas escolas pedagógicas.

Palavras-Chave: A formação Inicial de Pesquisa, Abordagem de Pesquisa, Professor Primário de Nível Médio, Ambiente Rural. 


\section{Introducción}

El proceso de formación de maestros en Cuba transita por una etapa de perfeccionamiento en sus planes de estudio, motivado por la necesidad de responder acertadamente a las demandas sociales que generan los acelerados cambios científicos tecnológicos que se despliegan. Las diferentes modalidades que se desarrollan en el país intentan reorganizar sus programas de estudio de manera que sus egresados sean capaces de cumplir su rol social eficientemente.

En la formación de maestros primarios de nivel medio que se desarrolla en las escuelas pedagógicas descansa la mayor cobertura docente que asume el proceso de enseñanza- aprendizaje de los centros primarios del país. De ahí la necesidad de elevar los resultados de esta concepción curricular, lo que deviene en calidad del proceso educativo. Estos estudiantes reciben una preparación general que abarca la dirección del proceso educativo, haciendo énfasis en el enfoque desarrollador. Al abordar las funciones profesionales pedagógicas: docente metodológica y de orientación se tiene en cuenta las características de los escenarios docentes en que se insertarán estos graduados.

Sin embargo, se presentan limitaciones para el abordaje de la investigación educativa como parte orgánica de esta preparación. En el estudio de los documentos rectores (perfil del egresado y orientaciones metodológicas) que rigen el proceso de formación de maestros primarios de nivel medio se constató que la investigación está explícitamente declarada como un elemento que se trabaja a modo de familiarización. Igualmente se certifica que la formación inicial investigativa del futuro maestro se concreta mediante diferentes formas de organización del proceso de enseñanza-aprendizaje. En estos documentos no se precisa el cómo, ni el qué de la investigación educativa que se abordará como parte de la carrera.

Este aspecto toma mayor trascendencia a partir de las exigencias expresadas en el Reglamento del Trabajo Metodológico en el Ministerio de Educación (MINED) en donde se prescribe en su artículo 2 la necesidad de elevar la calidad del proceso educativo mediante el perfeccionamiento constante de su desempeño profesional científico y creativo sobre la base de actuaciones éticas en correspondencia con la tradición pedagógica cubana y la cultura universal. Por ello debe ser capaz de asimilar los nuevos desafíos que van apareciendo sin afectar la calidad del proceso que dirige, lo 
que se traduce en satisfacción para estudiantes y padres.

En la bibliografía consultada se identifican autores que han profundizado en el estudio de la investigación educativa como parte de la formación pedagógica para el nivel universitario: Addine (1996) aborda la organización de la práctica laboral investigativa, Pérez (1997) presenta presupuestos para una reforma educativa que contiene elementos de la investigación; López (2001) profundizó en las habilidades investigativas para el profesor de Química, Salazar (2001) aportó en la formación interdisciplinaria del profesor de Biología, Chirino (2002) define la formación inicial investigativa para el profesional de la educación, Deler (2006) contextualiza la dirección de la actividad científica a la escuela primaria, Escalona (2008) enfatiza en la introducción de resultados como parte de la actividad científica, García (2011) presenta los principios, regularidades y competencias de la formación investigativa, y Vázquez (2014) profundiza en la actividad científico investigativa para el psicopedagogo, entre otros.

Desde la teoría hay limitaciones en el tratamiento de la formación investigativa para el nivel medio. Lo que no quiere decir que algunos de los aportes hechos por estos autores no puedan ser contextualizados a este nivel. Se considera que falta intencionalidad en el abordaje de los contenidos de la investigación educativa en la formación de nivel medio, Puesto que la preparación de estos maestros también tiene que responder a las exigencias de su práctica pre-profesional. Este nivel debe crear las premisas necesarias para su posterior enriquecimiento y profundización.

García (2011) plantea que la investigación convierte al educador en "sujeto de transformación social dedicado a la creación de una actitud de cambio en la escuela." (p. 2). Ello lo capacita para interpretar y trasformar la realidad educativa, identificar contradicciones en los diferentes escenarios y buscar soluciones científicas a estos problemas. Sobre esta base se considera que los nuevos contextos en que se desenvuelven los educadores de nivel medio imponen preparaciones con una base científico investigativa, que les permita interactuar en su realidad educativa y contribuir a su transformación creadora, por lo que se convierte en una necesidad en el proceso formativo de este profesional.

Sobre la base de lo anterior se pregunta ¿Qué tan eficiente será esta preparación para un egresado que se desempeñe como maestro primario rural? 
Las escuelas rurales en Cuba se muestran como una conquista social que no solo eleva la calidad de vida de las personas que viven en lugares de difícil acceso, sino que además materializa la voluntad política del país de eliminar las diferencias entre el campo y la ciudad. Son priorizadas en visitas de asesoramiento metodológico para garantizar la calidad del proceso educativo y en aseguramiento material.

Se comparte la idea de que "en el mundo se habla de una nueva ruralidad, desde la realidad del desarrollo científico técnico, económico y social; en Cuba, también se construye una ruralidad con características propias." (ECURED, 2016, p. 1) donde la Escuela Rural aparece como un faro luminoso de sabiduría y cultura. En este escenario está el maestro primario con sus estudiantes y padres brindando conocimiento, con sus manos colmadas de sueños y vocación para enseñar a sus discípulos el maravilloso oficio de encontrarse a sí mismos. (S/A, 2016, p. 1).

Esta bella labor está cargada de desafíos, pues el entorno le impone desventajas que deben eliminar o minimizar. Dentro de ellas:

Al ser un joven graduado presenta dudas ante situaciones que se presentan y no siempre cuenta con un tutor o consultante cerca que lo oriente.
La inasistencia de sus estudiantes en épocas de lluvia provoca atrasos en el programa de estudio.

- Las distancias que deben recorrer sus estudiantes diariamente para llegar a la escuela, provoca cansancio físico y espiritual para afrontar la tarea escolar del horario de la tarde fundamentalmente.

- La atención simultánea a varios grados que no siempre son consecutivos (aulas multigradas) que demandan mayor preparación para conducir adecuadamente el proceso de enseñanza-aprendizaje.

Estas debilidades hacen que la preparación del maestro primario rural se intenciones con conocimientos flexibles que les permitan dirigir el proceso de enseñanza-aprendizaje bajo una concepción desarrolladora, según lo exige el Modelo de la Escuela Primaria y que además pueda darle respuesta a los problema de su práctica laboral. El cambio en los contextos sociales exige nuevas respuestas desde el punto de vista de la ciencia. El maestro primario egresado de nivel medio de las escuelas pedagógicas que laborará en escuelas rurales multiplica la necesidad de apropiarse de los contenidos de la investigación educativa, lo que le permitirá estar a la altura de su entorno. 
Sobre esta base, ¿Cómo potenciar la formación del maestro primario de nivel medio que se desempeñará en escuelas rurales?

Por consiguiente se plantea como objetivo de este artículo: reflexionar acerca de las posibilidades que ofrece el enfoque investigativo para el abordaje de los contenidos de la investigación educativa en el proceso de formación inicial de los maestro primario de nivel medio que se desempeñará en escuelas rurales.

\section{Desarrollo}

A partir del año 2000, aparecen nuevas expectativas para el nivel primario con el surgimiento del Modelo de Escuela primaria, este se instrumentó en la totalidad de los centros y se distingue, entre otras, por las siguientes características:

El aprendizaje que se concibe a partir de ese Modelo contribuye a una formación integral de la personalidad del escolar primario. Se proyecta la organización escolar como punto de partida, donde todos los factores y agentes del centro escolar eduquen, en el que cada uno tiene sus funciones bien definidas. Cada docente atiende un grupo de hasta veinte alumnos de un mismo grado, apoyándose en los medios audiovisuales que la Revolución ha puesto a su disposición $\mathrm{y}$ que existen en todas las escuelas primarias del país. (ECURED, 2016, p.4)

Estas exigencias demandaron de estudio, profundización e intercambios profesionales de los maestros primarios, con énfasis, los que laboran en zonas rurales para la implementación del modelo. La escuela rural por las características de los asentamientos poblacionales, es el centro institucional alrededor del cual gira el desarrollo personal del niño en contacto inseparable con su comunidad, así como de padres y vecinos. Su dinámica involucra a todos en el proceso educacional, por ello la importancia de la preparación del docente que se desempeña en este escenario.

Se concuerda con los autores Rodríguez, Rodríguez y Rubié (2015) quienes plantean que "la preparación del maestro tiene como objetivo la formación permanente y la actualización sistemática de sus conocimientos, el perfeccionamiento del desempeño de sus actividades profesionales y académicas así como el enriquecimiento de su acervo cultural." (p. 88) Para el cumplimiento de este fin es necesario potenciar la apropiación de los conocimientos de la investigación educativa como función profesional pedagógica.

Esta función nos adentra en el cómo actuar ante tantos problemas sociales y a su vez, tantos avances tecnológicos que generan un gran volumen de conocimientos. "La investigación pasa, por tanto a ser un proceso asociado a la construcción del conocimiento definiéndose 
más como un propósito, como un objetivo del profesional, que como una actividad particular, y se convierte así en un modo de actuación profesional.” (Salazar, 2001, p. 43).

En esta línea de análisis se hace necesario precisar qué se entiende por investigación educativa y la autora asume la definición de Chirino Ramos quien la declara como "el proceso de búsqueda, mediante el método científico, de conocimientos esenciales en el área de la educación que aporten alternativas de solución a los problemas pedagógicos." (Chirino, 2002, p. 1). Este paso de búsqueda y aporte de soluciones como parte de la formación inicial investigativa es un proceso continuo donde se desarrollan habilidades, capacidades y valores de la actividad científica, que constituyen contenido de la formación y el perfeccionamiento en la preparación del profesional.

Entendemos por formación inicial investigativa para los maestros primarios de nivel medio el proceso de apropiación de los contenidos mínimos indispensables y flexibles desde un enfoque investigativo que sustente el ejercicio de la función investigativa en su desempeño profesional.

Se identifican los contenidos como mínimos indispensables, pues este nivel constituye un primer paso en el gran proceso de formación permanente de un docente, flexibles pues en el campo de la ciencia la teoría no es acabada y está en constante cambio, lo que muestra la dialéctica de la evolución humana. Un enfoque investigativo que guíe el currículo de estudio en la profundización, integración y reforzamiento de los conocimientos de las asignaturas que lo conforman. La formación investigativa debe ser un proceso que contenga el estudio del método científico y sus etapas, así como otros de carácter teórico y empírico de la investigación educativa necesaria para su desempeño. Es además, sistémico en tanto mantiene estrecha relación con los restantes componentes del proceso.

Como parte de la lógica que se expone, es necesario profundizar en el enfoque investigativo para la formación de maestros primarios de nivel medio. Se aprecia como la estrategia general del proceso de enseñanza-aprendizaje que asegura el desarrollo de conocimientos, hábitos, habilidades y valores profesionales pedagógicos que sustentan la función investigativa, a partir de la estructuración, sistematización e integración de las asignaturas del currículo de estudio que realza las características del modelo del profesional y crea las bases para el desarrollo de un modo de actuación 
en correspondencia con las exigencias actuales.

El ideal de maestro primario de nivel medio debe responder al Modelo General del Profesional de la Educación; por lo que debe materializar que "la formación en la práctica como fuente de necesidades y problemas profesionales, privilegia los modos de actuación que se transforman en eje articulador del currículo y se convierte, a su vez en estrategia de aprendizaje desarrollador." (Chirino, 2002, p. 29), lo que hace a los futuros docentes partícipes de su formación y aumenta el sentido de pertenencia con la profesión. El profundizar en el escenario rural hace que las problemáticas que se identifican en él sean abordadas como parte del conocimiento con una visión problematizadora y transformadora.

Se considera además que crea las bases para el desarrollo de un modo de actuación, que:

Si bien no llega a desarrollar las capacidades pedagógicas a plenitud, sí debe alcanzar un desarrollo de los conocimientos científicos, las habilidades y valores profesionales que sientan las bases para las mismas, demostrando niveles cada vez superiores de creatividad en su desempeño. (Chirino, 2002, p. 29).

El enfoque investigativo en el proceso de formación de maestros primarios de nivel medio crea condiciones objetivas para el mejoramiento de la calidad del trabajo educacional, con énfasis en los que se desempeñará en escuelas rurales, con ello aumenta el reconocimiento social a su labor pedagógica. Se considera que el sistematizar el enfoque investigativo en la formación de maestros primarios de nivel medio presupone:

\section{- Una conexión directa entre} la formación del futuro docente y su medio de realización.

$$
\text { - Un antecedente al }
$$
componente investigativo de la formación universitaria que crea condiciones objetivas para profundizar en la esencia de la investigación educativa.

$$
\text { - Una mayor vinculación }
$$

entre la teoría contextualizada y la práctica problematizadora como principio básico de la formación.

\section{Se considera que lo más prudente no es entretenerse en abstracciones, sino en hacer práctica las posiciones teóricas útiles, como dijera el Apóstol cubano. En este caso es bueno pronunciarse por un enfoque investigativo que exprese la real unidad entre lo empírico y lo teórico, lo cuantitativo y lo cualitativo, sin que tenga que acogerse a una $u$ otra tendencia. (Chávez, 2010, p. 176).}

\section{- La intensificación de} acciones que enfatizan el enfoque profesional pedagógico de la carrera. 
Se hace necesario exponer las características del enfoque investigativo en la formación de maestros primarios de nivel medio, lo que implicar reconocer los elementos que lo distinguen y que avalan su singularidad:

- Carácter sistémico a partir de integral en una misma dirección los objetivos y contenidos de las asignaturas, con los de la investigación en estrechas relaciones internas que benefician la adquisición de independencia cognitiva en la búsqueda de información, la lectura crítica, la elaboración de resúmenes y el desarrollo del interés por la investigación como vía científica para resolver sus problemáticas.

- Carácter problematizador que indica las características el proceso de enseñanza y aprendizaje a desarrollar, que impone la presentación de gran parte del contenido como tareas productivas que el educando resuelve investigando e intercambiando opiniones con los miembros de su aula y docentes del centro donde realiza su práctica; predomina la propuesta de hipótesis, ideas científicas y/o estrategias de aprendizaje aportadas por los estudiantes para la solución a sus inquietudes, lo que muestra una implicación directa en su formación e introduce cambios en el sistema evaluativo, pues no sólo se tendrá en cuenta en las evaluaciones sistemáticas de trabajos investigativos (RM 200/2014) sino que su alcance llegará hasta la evaluación de su práctica docente y la culminación de estudios de la carrera.

\section{- Carácter transformador} pues evoluciona la dinámica tradicional del aula y la práctica preprofesional al reducir el trabajo frontal del profesor y la consulta con el tutor. Aumenta el tiempo de trabajo independiente y de reflexión de los educandos sobre las contradicciones entre lo conocido y lo desconocido en las materias de estudio y su realidad educativa; por lo que asume una actitud reflexiva permanente en su formación.

- Carácter participativo en tanto involucra estudiantes y profesores en intercambios de socialización con sus compañeros y docentes de su práctica, pues expone, discrepa y defiende opiniones que sustentan sus soluciones e ideas. Recibe experiencias e información que retroalimentan su preparación y favorece la toma de decisiones. Se siente, es y toma parte de su proceso de formación.

\section{- Carácter metodológico a} partir de tener como esencia el trabajo 
con el método científico para el desarrollo de habilidades científico investigativas. (problematizar, teorizar y comprobar) que concretan la función investigativa del maestro primario de nivel medio, lo que presupone la orientación desde las clases de actividades investigativas para la práctica pre-profesional.

- Carácter estratégico en tanto condiciona los antecedentes necesarios para la materialización de las funciones profesionales en un modo de actuación que se perfeccionará como parte del desempeño profesional del maestro primario del nivel medio.

- Carácter flexible y diferenciado se materializa en la habilidad de seleccionar de las diferentes asignaturas de los años de la carrera los contenidos que propician trabajar con el método científico. Su selección y concepción responde al diagnóstico de estudiantes y al contexto de la práctica docente. Poniendo como centro las problemáticas de la institución, la familia y/o la comunidad que afectan sus resultados de trabajo.

El enfoque investigativo convierte el conocimiento en una necesidad para el desempeño eficientemente de los futuros maestros, los transforma en protagonista de su propia formación con una visión dialéctica, flexible, y transformadora de su misión social. Convierte el aula en un espacio de intercambio científico pedagógico que socializa y divulga experiencias investigativas y que nos prepara para la presentación y discusión de resultados científicos en eventos y otros escenarios.

\begin{abstract}
Se puede y se debe convertir al docente en un investigador científico. Este particular redundará sin dudas, en la consolidación de su formación académica, técnica y en la segura elevación de la calidad del proceso de enseñanza - aprendizaje. Así se trabaja a favor de la calidad desde la propia masividad. (Chávez, 2010, p. 177-178).
\end{abstract}

Estas características destacan en la formación de maestros primarios de nivel medio nexos importantes que muestran las conexiones que se establecen entre los componentes del proceso. El enfoque investigativo enfatiza las relaciones entre:

$$
\text { - Las funciones del }
$$

profesional de la educación al atravesarlas como eje transversal para su desarrollo.

\section{- La formación investigativa y} el proceso de enseñanza y aprendizaje (PEA) desarrollador que se aplica en el modelo de la escuela primaria actual.

La teoría aprendida y la práctica pedagógica al desarrollar un método de cuestionamiento y experimentación 
permanente que con sus respuestas enriquece su desempeño profesional.

- Las asignaturas del currículo y el modelo del profesional que se necesita formar, desde una dialéctica flexible que permite introducir los nuevos cambios de los escenarios escolares como expresión de actualización permanente.

\section{- La formación de maestros} primarios de nivel medio y la universitaria al convertirse en antecedente del componente investigativo.

Por todo lo expuesto, se sustenta que se debe asumir el enfoque investigativo en la formación de maestros primarios de nivel medio, con énfasis en los que se desempeñará en escuelas rurales; pues favorece que los contenidos de la investigación educativa constituyan eje transversal de las asignaturas para que el estudiante la desarrolle como función profesional en interrelación con las restantes funciones. Ello impulsará el modo de actuación en correspondencia con los cambios que requiere la escuela primaria actual y contribuye al perfeccionamiento del proceso docente educativo que se desarrolla en los centros formadores, a partir del desarrollo de una concepción científica del mundo y un método científico de trabajo en el docente. ¿Cómo crear actividades con enfoque investigativo?
Concebir actividades con enfoque investigativo en las diferentes asignaturas implica identificar los problemas de la realidad educativa que se relacionan con la misma, por lo que hay que problematizar dicha realidad haciendo evidentes las contradicciones esenciales que afectan la calidad del proceso. Para ello se pueden utilizar métodos de carácter empíricos que permiten la recopilación de información como: observación, entrevistas, encuestas y el cuestionario.

La presentación de los resultados de estos instrumentos puede ser en debates grupales o en exposiciones organizadas para este efecto. Con el planteamiento de los problemas a resolver, el conocimiento se presenta como alternativa de solución a los mismos. Ello conduce a organizarlos con una lógica que favorezca ir de lo simple a lo complejo y relacionándolos como parte de un mismo sistema. Este proceso es parte de la teorización del contenido, que además se enriquece con otros métodos de carácter teóricos que permiten el intercambio con otras fuentes bibliográficas y otros puntos de vista. Los programas de las asignaturas se respetan, solo se reorganiza el contenido de manera que realce la vinculación entre la práctica educativa y la teoría científica pedagógica.

Esta concepción beneficia la relación entre los diferentes escenarios de 
formación, pues después de haber gestionado el conocimiento, los estudiantes deben identificar cuál o cuáles le ayudan a resolver su problemática, diseñar acciones que permitan su erradicación o disminución y llevarlo a su práctica laboral. En este momento se comprueba la eficiencia de la propuesta y se rediseña según los resultados obtenidos.

Este proceso aumenta el significado del contenido y su alcance en la solución a los problemas de su realidad educativa. Para la comunicación de estos resultados se pueden organizar seminarios docentes y/o pueden ser presentados en eventos de carácter extra docentes que acentúan la formación investigativa. Estos espacios potencian la confrontación de criterios, la fundamentación de sus puntos de vistas y el respeto científico a posiciones diferentes.

Se concuerda con Chirino cuando expresa que "la evaluación sistemática se basa fundamentalmente en la exposición y debate científico de los fundamentos teóricos que van extrayendo de los textos consultados, y las posiciones asumidas al respecto, durante las sesiones científicas." (Chirino, 2002, p. 95).

Como resumen en el proceso docente se deben organizar actividades que les permitan a los estudiantes:
- Identificar problemas de la realidad educativa.

- Realizar análisis teórico del problema identificado a partir del conocimiento gestionado y otras fuentes de consulta.

- Elaborar instrumentos investigativos, aplicarlos y procesarlos.

- Diseñar acciones que conformen alternativas de solución al problema investigado.

- Elaborar el informe del trabajo investigativo.

Del escenario rural donde se desarrolla la práctica pedagógica de este egresado se extraen las contradicciones que afectan la obtención de los resultados esperados, se verifica la viabilidad de las soluciones propuestas y en él se manifiesta el modo de actuación profesional que queremos formar. Por tanto, las actividades de su práctica laboral deben estar bien orientadas, de ahí la importancia de las guías de la práctica docente que se elaboran por años de la carrera.

La guía de la práctica docente debe caracterizarse por la definición clara de los objetivos a lograr, los contenidos que se necesitan para ello, así como los métodos y medios a utilizar. Por tanto, este instrumento concreta un sistema de tareas de carácter independiente, fundamentalmente, que conlleva a ir 
desarrollando un modo de actuación profesional reflexivo, crítico y emprendedor. Lo anterior, demanda que estas estén conectadas como parte de un mismo sistema, graduadas y evaluadas con la misma concepción. El cumplimiento de estas actividades en el contexto rural se dimensiona en tanto aumenta el carácter científico del proceso y logra desarrollar en sus estudiantes métodos de estudio y de actualización al nivel de las zonas urbanas.

La evaluación del cumplimiento de estas tareas deviene en valoración del desempeño de los maestros primarios de nivel medio y favorece también valuar la formación inicial investigativa de estos docentes.

Esta estrategia para el maestro primario que se despeñará en escuelas rurales lo adentra en las problemáticas más significativa de su entorno, le crea un estilo de trabajo participativo $y$ desarrollador que favorece el trabajo comunitario en y desde la escuela, le permite ajustar su proceso educativo a sus características, reorganizar sus contenidos y con todo ello ser más eficiente en su desempeño profesional.

\section{Conclusiones}

- El maestro primario rural necesita de una formación inicial investigativa que le permita apropiarse de los contenidos mínimos indispensables y flexibles desde un enfoque investigativo que le sustente el ejercicio de la función investigativa en su desempeño profesional.

- El enfoque investigativo por su esencia constituye germen de formación, y génesis de un modo de actuación profesional trasformador que le impregna un profundo carácter humanista al proceso formativo de maestros primarios de nivel medio. Produce un tránsito hacia niveles superiores de desarrollo de los estudiantes, materializado en el progreso de una actitud crítica, un pensamiento científico y un desempeño exitoso en el mejoramiento del contexto de actuación pedagógica.

\section{Referencias}

Addine, F. (1996). Alternativa para la organización de la práctica laboral investigativa en los Institutos Superiores Pedagógicos. ISP "EJV". (Tesis de Grado Científico en Ciencias Pedagógicas). La Habana.

Centro de Estudios Educacionales. (2000). Modelo General del Profesional de la Educación. Glosario de términos. Instituto Superior Pedagógico "Enrique José Varona, La Habana, Cuba.

Chávez J. (2010). La investigación científica desde la escuela. Modulo II Fundamentos de las Ciencias de la Educación. Maestría en Ciencias de la Educación. Ira parte. (pp. 174- 179). La Habana, Cuba Editorial Pueblo y educación. 
Chirino, M V. (2002) Perfeccionamiento de la formación inicial investigativa de los profesionales de la educación. (Tesis en opción al Grado Científico de Doctor en Ciencias Pedagógicas). La Habana, Cuba. Instituto Superior Pedagógico "Enrique José Varona".

Deler, G. (2006). Estrategia para la dirección de la actividad científicoinvestigativa del docente en el centro de referencia de la educación primaria. (Tesis presentada en opción al Grado Científico de Doctor en Ciencias Pedagógicas). Instituto Central de Ciencias Pedagógicas.

ECURED. (2016) Escuela rural cubana. Consultado martes, 13 de septiembre en: file:///D:/Escuelas\%20rurales/Escuela\%20r ural\%20cubana\%20-\%20EcuRed.html

Escalona, E. (2008). Estrategia de introducción de resultados de investigación en el ámbito de la actividad científica educacional. (Tesis de Doctorado). Instituto Central de Ciencias Pedagógicas.

Faleiro, W., \& Farias, M. N. (2016). Formadores de professores em Educação do Campo em Goiás. Rev. Bras. Educ. Camp., 1(1), 88-106.

García, G. (2011). Enfoque interdisciplinario de la investigación educativa desde la dialéctica materialista. La formación investigativa. Investigación interdisciplinaria en las ciencias pedagógicas. (pp. 1 - 24). La Habana. Cuba. Editorial Pueblo y Educación.

MINED. Planes de estudio de la especialidad de Maestros Primarios de nivel medio. 2010-2011, 2011-2012 y 2012-2013. Soporte digital.

MINED (2011). Formación de maestros primarios de nivel medio superior. Soporte digital.
MINED (2014). Resolución Ministerial 200. Reglamento del Trabajo Metodológico. Empresa poligráfica de Holguín, ARGRAF. Registro n 023-2013.

MINED. (S/A) Perfil del egresado de media superior. Soporte digital

Las escuelas rurales. Generalidades III. (2016) Consultado el día 19 de septiembre en:

file:///D:/Escuelas\%20rurales/GENERALI DADES\%20III_files/GENERALIDADES \%20III.htm

López, L. (2001). El desarrollo de habilidades investigativas en los estudiantes de la Licenciatura en Química. (Tesis de Doctorado). Universidad de Cienfuegos "Carlos Rafael Rodríguez”.

Pérez, A. I. (1997). Historia de una Reforma Educativa. Díada Editora, Sevilla, España

Rico M., Bonet C., Castillo S., García O., Martín-Viaña C., Rizo C. y Santos P. (2000). Hacia el perfeccionamiento de la escuela Primaria. La Habana, Cuba. Editorial Pueblo y Educación.

Rodríguez R. F., Rodríguez J. C., \& Rubié A. G. (2015). La preparación a maestros de escuelas rurales multigrados, una propuesta de talleres metodológicos para fortalecer la educación ambiental. Revista IPLAC, (6), 83-95.

Salazar D, (2001). La formación interdisciplinaria del futuro profesor de Biología en la actividad científica investigativa. (Tesis de Doctorado). Ciudad de La Habana.

Vázquez D. (2014) Concepción didáctica para la dirección de la actividad científico-investigativa en la formación inicial profesional del psicopedagogo. (Tesis de Doctorado). La Habana. 
Recebido em: 18/10/2016

Aprovado em: 25/10/2016

Publicado em: 19/04/2017

Como citar este artigo / How to cite this article / Como citar este artículo:

APA:

Grandal, M. L. R. (2017). La formación del maestro primario rural. Rev. Bras. Educ. Camp., 2(1), 07-22. DOI: $\quad$ http://dx.doi.org/10.20873/uft.2525$\underline{4863.2017 \mathrm{v} 2 \mathrm{n} 1 \mathrm{p} 07}$

ABNT:

GRANDAL, M. L. R. La formación del maestro primario rural. Rev. Bras. Educ. Camp., Tocantinópolis, v. 2, n. 1, p. 07-22, 2017. DOI: http://dx.doi.org/10.20873/uft.2525-

4863.2017v2n1p07 